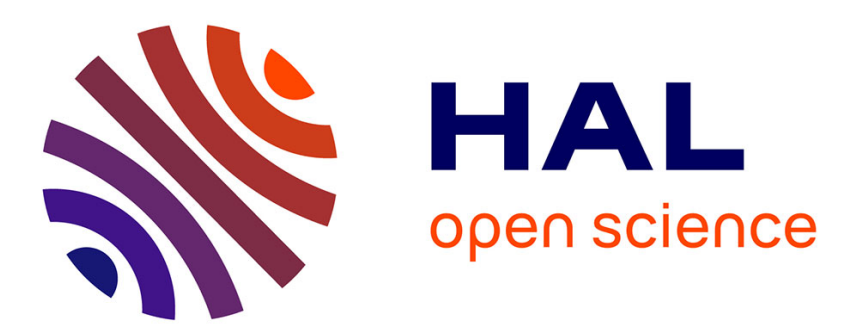

\title{
Semi-global minimal time hybrid robust stabilization of analytic driftless control-affine systems
}

Christophe Prieur, Emmanuel Trélat

\section{To cite this version:}

Christophe Prieur, Emmanuel Trélat. Semi-global minimal time hybrid robust stabilization of analytic driftless control-affine systems. 2005, 6 p. hal-00086462

\section{HAL Id: hal-00086462 https://hal.science/hal-00086462}

Submitted on 18 Jul 2006

HAL is a multi-disciplinary open access archive for the deposit and dissemination of scientific research documents, whether they are published or not. The documents may come from teaching and research institutions in France or abroad, or from public or private research centers.
L'archive ouverte pluridisciplinaire HAL, est destinée au dépôt et à la diffusion de documents scientifiques de niveau recherche, publiés ou non, émanant des établissements d'enseignement et de recherche français ou étrangers, des laboratoires publics ou privés. 


\title{
Semi-global minimal time hybrid robust stabilization of analytic driftless control-affine systems
}

\author{
Christophe Prieur and Emmanuel Trélat
}

\begin{abstract}
We investigate the problem of semi-global minimal time robust stabilization of analytic driftless control-affine systems, by means of a hybrid state feedback law. Our main result is that, in the absence of singular minimal time solutions, the solutions of the closed-loop system converge to the origin in quasi-minimal time (for a given bound on the controller) with a robustness property with respect to small measurement noise and external disturbances.
\end{abstract}

\section{INTRODUCTION}

Let $m$ and $n$ be two positive integers. Consider on $\mathbb{R}^{n}$ the driftless control-affine system

$$
\dot{x}(t)=\sum_{i=1}^{m} u_{i}(t) f_{i}(x(t))
$$

where $f_{1}, \ldots, f_{m}$ are analytic vector fields on $\mathbb{R}^{n}$, and where the control function $u(\cdot)=\left(u_{1}(\cdot), \ldots, u_{m}(\cdot)\right)$ satisfies the constraint

$$
\sum_{i=1}^{m} u_{i}(t)^{2} \leq 1
$$

The system (1), together with the constraint (2), is said to be globally asymptotically stabilizable, if, for each point $x \in$ $\mathbb{R}^{n}$, there exists a control law satisfying the constraint (2) such that the solution of (1) associated to this control law and starting from $x$ tends to 0 as $t$ tends to $+\infty$, and satisfies a stability property (see [36] e.g. for a precise statement).

This asymptotic stabilization problem has a long history and has been widely investigated. Note that, due to Brockett's condition [10, Theorem 1, (iii)], if $m<n$ then there does not exist any continuous stabilizing feedback law for (1). However several control laws have been derived for such control systems (see for instance [24], [21], [5] and references therein).

The robust asymptotic stabilization problem is under current and active research. Many notions of controllers have been introduced to treat this problem, such as discontinuous sampling feedbacks [13], [35], time varying control laws [15], [14], [25], [26], patchy feedbacks (as in [4]), SRS feedbacks [34], ..., enjoying different robustness properties depending on the errors under consideration.

We consider here feedback laws having both discrete and continuous components, which generate closed-loop systems with hybrid terms (see for instance [39]). Such feedbacks

C. Prieur is with LAAS-CNRS, 7, avenue du Colonel Roche 31077 Toulouse, France christodhe.priernlaas.fr

C. Prieur's research is partly done in the framework of the HYCON Network of Excellence, contract number FP6-IST-511368.

E. Trélat is with Univ. Paris-Sud, Labo. AN-EDP, Bat. 425, 91405 Orsay Cedex, France emanuel.trelatcrath.u-psud.fr appeared first in [28] to stabilize nonlinear systems having a priori no discrete state. They consist in defining a switching strategy between several smooth control laws defined on a partition of the state space.

It can be now seen as a paradigm for the robust stabilization of nonlinear control systems for which we are not able (because of the topological obstructions), or we do not know how to compute a continuous or a discontinuous stabilizing feedback. This strategy appears in [28] and has been used, among others, in [30], [29], [18], [33]. It requires a precise statement of the notion of a solution, and of the notion of asymptotically stable systems, as studied e.g. in [29], [18].

Here we use this strategy together with an optimal objective, focusing on the robust stabilization property and on the optimality of the speed of convergence. It requires a precise study of the minimal time control problem of (1) under the constraint (2). We use a hybrid strategy to unit different control laws. This allows to switch between the components of the hybrid feedback law by guaranteeing a robustness property with respect to measurement noises and external disturbances.

More precisely, in a first step, we consider the minimal time problem for the system (1) with the constraint (2), of steering a point $x_{0} \in \mathbb{R}^{n}$ to the origin. Note that this problem is solvable as soon as Hörmander's condition is satisfied on the $m$-tuple of vector fields $\left(f_{1}, \ldots, f_{m}\right)$. Of course, in general it is impossible to compute explicitly the optimal time feedback controllers for this problem. Moreover, Brockett's condition implies that such control laws are not smooth whenever $m<n$ and the vector fields $f_{1}, \ldots, f_{m}$ are independent. This raises the problem of the regularity of optimal feedback laws. The literature on this subject is immense. In an analytic setting, the problem of determining the analytic regularity of the value function for a given optimal control problem, has been, among others, investigated by [37]. For systems of the form (1), the minimal time problem under the constraint (2) is equivalent to the sub-Riemannian problem associated to the $m$-tuple of vector fields $\left(f_{1}, \ldots, f_{m}\right)$. In this framework, the minimal time function to $x_{0}$ is equal to the sub-Riemannian distance to $x_{0}$. The analytic regularity of the sub-Riemannian distance is related to the existence of singular minimizing solutions, see [1], [2], [40]. More precisely, if there does not exist any nontrivial singular minimizing solution starting from 0 , then the sub-Riemannian distance to 0 is subanalytic outside 0 (see [19], [20] for a general definition of subanalytic sets). In particular, this function is analytic outside a stratified submanifold $\mathcal{S}$ of $\mathbb{R}^{n}$, of codimension greater than or equal 
to 1 , see [38]. As a consequence, outside this submanifold it is possible to provide an analytic optimal time feedback controller for the system (1) with the constraint (2).

Note that the analytic context is used so as to ensure stratification properties, which do not hold a priori if the system is smooth only. These properties are related to the notion of o-minimal category (see [16]).

Then, in a second step, by assuming the Hörmander's condition, we have to define a suitable feedback law such that all solutions go out of a given neighborhood of $\mathcal{S}$ within a small fixed time.

Finally, in order to achieve a minimal time robust stabilization procedure, using a hybrid feedback law (more precisely, a hysteresis), we unit the three feedback law-components. This part of our work is not new and analogous to [33], however we can not apply the robustness result of [33] directly, since, here, we are interested in a quasi-optimal property.

We thus give an alternative solution to a conjecture of $[9$, Conj. 1, p. 101], in which the existence of patchy feedbacks is conjectured (this is however a different matter).

In a previous paper [31], this program was achieved explicitly on the so-called Brockett system, for which $n=3$, $m=2$, and, denoting $x=\left(x_{1}, x_{2}, x_{3}\right)$,

$$
f_{1}=\frac{\partial}{\partial x_{1}}+x_{2} \frac{\partial}{\partial x_{3}}, f_{2}=\frac{\partial}{\partial x_{2}}-x_{1} \frac{\partial}{\partial x_{3}} .
$$

In this case, there does not exist any nontrivial singular solution, and the manifold $\mathcal{S}$ is the axis $\left(0 x_{3}\right)$.

\section{DEFINITIONS AND MAIN RESUlT}

\section{A. The minimal time problem}

Consider the minimal time problem for the system (1) with the constraint (2).

Definition 2.1: We say that Hörmander's condition holds if the Lie algebra spanned by the vector fields $f_{1}, \ldots, f_{m}$, is equal to $\mathbb{R}^{n}$ at every point $x$ of $\mathbb{R}^{n}$.

It is well-known that under this condition, any two points of $\mathbb{R}^{n}$ can be joined by a minimal time solution of (1), (2).

We denote by $T\left(x_{0}\right)$ the minimal time needed to steer the system (1) with the constraint (2) from a point $x_{0} \in \mathbb{R}^{n}$ to the origin.

Remark 2.1: Obviously, the control function $u: \mathbb{R}^{n} \rightarrow$ $\mathbb{R}^{m}$ associated to a minimal time solution of (1), (2), actually satisfies $\sum_{i=1}^{m} u_{i}^{2}=1$.

For $T>0$, let $\mathcal{U}_{T}$ denote the (open) subset of $u($.$) in$ $L^{\infty}\left([0, T], \mathbb{R}^{m}\right)$ such that the solution of (1), starting from 0 and associated to a control $u(\cdot) \in \mathcal{U}_{T}$, is well defined on $[0, T]$. The mapping

$$
\begin{aligned}
E_{T}: \mathcal{U}_{T} & \longrightarrow \mathbb{R}^{n} \\
u(\cdot) & \longmapsto x(T),
\end{aligned}
$$

which to a control $u(\cdot)$ associates the end-point $x(T)$ of the corresponding solution $x(\cdot)$ of (1) starting at 0 , is called end-point mapping at the origin, in time $T$; it is a smooth mapping.
Definition 2.2: A solution $x(\cdot)$ of system (1), so that $x(0)=0$, is said to be singular on $[0, T]$ if its associated control $u(\cdot)$ is a singular point of the end-point mapping $E_{T}$ (i.e. if the Fréchet derivative of $E_{T}$ at $u(\cdot)$ is not onto).

Remark 2.2: If $x(\cdot)$ is singular on $[0, T]$, then it is singular on $[0, t]$, for every $t \in(0, T)$.

\section{B. Class of controllers and notion of hybrid solution}

Let $f: \mathbb{R}^{n} \times \mathbb{R}^{m} \rightarrow \mathbb{R}^{n}$ be defined by $f(x, u)=$ $\sum_{i=1}^{m} u_{i} f_{i}(x)$. The system (1) writes

$$
\dot{x}(t)=f(x(t), u(t)) \text {. }
$$

The controllers under consideration depend on the continuous state $x \in \mathbb{R}^{n}$ and also on a discrete variable $s_{d} \in \mathcal{N}$, where $\mathcal{N}$ is a countable subset of $\mathbb{N}$. According to the concept of a hybrid system of [17], we introduce the following definition.

Definition 2.3: A hybrid feedback is a 4-tuple $\left(C, D, k, k_{d}\right)$, where

- $C$ and $D$ are subsets of $\mathbb{R}^{n} \times \mathcal{N}$;

- $k: \mathbb{R}^{n} \times \mathcal{N} \rightarrow \mathbb{R}^{m}$ is a function;

- $k_{d}: \mathbb{R}^{n} \times \mathcal{N} \rightarrow \mathcal{N}$ is a function.

The sets $C$ and $D$ are respectively called the controlled continuous evolution set and the controlled discrete evolution set.

We next recall the notion of robustness to small noises (see [36]). Consider two functions $e$ and $d$ satisfying the following regularity assumptions:

$$
\begin{aligned}
& e(\cdot, \cdot), d(\cdot, \cdot) \in L_{\text {loc }}^{\infty}\left(\mathbb{R}^{n} \times[0,+\infty) ; \mathbb{R}^{n}\right), \\
& e(\cdot, t), d(\cdot, t) \in C^{0}\left(\mathbb{R}^{n}, \mathbb{R}^{n}\right), \forall t \in[0,+\infty) .
\end{aligned}
$$

We introduce these functions as a measurement noise $e$ and an external disturbance $d$. Below, we define the perturbed hybrid system $\mathcal{H}_{(e, d)}$. The notion of solution of such hybrid perturbed systems has been well studied in the literature (see e.g. [7], [8], [23], [39], [29], [30]). Here, we consider the notion of solution given in [17], [18].

Definition 2.4: Let $S=\bigcup_{j=0}^{J-1}\left[t_{j}, t_{j+1}\right] \times\{j\}$, where $J \in$ $\mathbb{N} \cup\{+\infty\}$ and $\left(x_{0}, s_{0}\right) \in \mathbb{R}^{n} \times \mathcal{N}$. The domain $S$ is said to be a a hybrid time domain. A map $\left(x, s_{d}\right): S \rightarrow \mathbb{R}^{n} \times \mathcal{N}$ is said to be a solution of $\mathcal{H}_{(e, d)}$ with the initial condition $\left(x_{0}, s_{0}\right)$ if

- the map $x$ is continuous on $S$;

- for every $j, 0 \leq j \leq J-1$, the map $x: t \in\left(t_{j}, t_{j+1}\right) \mapsto$ $x(t, j)$ is absolutely continuous;

- for every $j, 0 \leq j \leq J-1$ and almost every $t \geq 0$, $(t, j) \in S$, we have

$$
\left(x(t, j)+e(x(t, j), t), s_{d}(t, j)\right) \in C,
$$

and

$$
\begin{aligned}
\dot{x}(t, j)= & f\left(x(t), k\left(x(t, j)+e(x(t, j), t), s_{d}(t, j)\right)\right) \\
& +d(x(t, j), t), \\
\dot{s}_{d}(t, j)= & 0
\end{aligned}
$$


(where the dot stands for the derivative with respect to the time variable $t$ )

- for every $(t, j) \in S,(t, j+1) \in S$, we have

$$
\left(x(t, j)+e(x(t, j), t), s_{d}(t, j)\right) \in D,
$$

and

$$
\begin{aligned}
x(t, j+1) & =x(t, j), \\
s_{d}(t, j+1) & =k_{d}\left(x(t, j)+e(x(t, j), t), s_{d}(t, j)\right) ;
\end{aligned}
$$

- $\left(x(0,0), s_{d}(0,0)\right)=\left(x_{0}, s_{0}\right)$.

In this context, we next define the concept of stabilization of (3) by a minimal time hybrid feedback law sharing a robustness property with respect to measurement noises and external disturbances. The usual Euclidean norm in $\mathbb{R}^{n}$ is denoted by $|\cdot|$. Recall that a function of class $\mathcal{K}_{\infty}$ is a function $\delta:[0,+\infty) \rightarrow[0,+\infty)$ which is continuous, increasing, satisfying $\delta(0)=0$ and $\lim _{R \rightarrow+\infty} \delta(R)=+\infty$.

Definition 2.5: Let $\rho: \mathbb{R}^{n} \rightarrow \mathbb{R}$ be a continuous function satisfying

$$
\rho(x)>0, \forall x \neq 0 .
$$

We say that the completeness assumption for $\rho$ holds if, for all $(e, d)$ satisfying the regularity assumptions (4), and so that,

$$
\begin{aligned}
& \sup _{[0,+\infty)}|e(x, \cdot)| \leq \rho(x), \forall x \in \mathbb{R}^{n}, \\
& \operatorname{esssup}_{[0,+\infty)}|d(x, \cdot)| \leq \rho(x), \forall x \in \mathbb{R}^{n},
\end{aligned}
$$

for every $\left(x_{0}, s_{0}\right) \in \mathbb{R}^{n} \times \mathcal{N}$, there exists a maximal solution on $[0,+\infty)$ of $\mathcal{H}_{(e, d)}$ starting from $\left(x_{0}, s_{0}\right)$.

Definition 2.6: We say that the uniform finite time convergence property holds if there exists a continuous function $\rho: \mathbb{R}^{n} \rightarrow \mathbb{R}$ satisfying (9), such that the completeness assumption for $\rho$ holds, and if there exists a function $\delta$ : $[0,+\infty) \rightarrow[0,+\infty)$ of class $\mathcal{K}_{\infty}$ such that, for every $R>0$, there exists $\tau=\tau(\operatorname{diam}(R))>0$, for all functions $e, d$ satisfying the regularity assumptions (4) and inequalities (10) for this function $\rho$, for every $x_{0} \in B(0, R)$, and every $s_{0} \in \mathcal{N}$, the maximal solution $\left(x, s_{d}\right)$ of $\mathcal{H}_{(e, d)}$ starting from $\left(x_{0}, s_{0}\right)$ satisfies

$$
|x(t, j)| \leq \delta(R), \forall t \geq 0,(t, j) \in S,
$$

and

$$
x(t, j)=0, \forall t \geq \tau,(t, j) \in S .
$$

Definition 2.7: The origin is said to be a semi-global minimal time hybrid robust stabilizable equilibrium for the system (3) if, for every $\varepsilon>0$ and every compact subset $K \subset \mathbb{R}^{n}$, there exists a hybrid feedback law $\left(C, D, k, k_{d}\right)$ satisfying the constraint

$$
\left\|k\left(x, s_{d}\right)\right\| \leq 1
$$

where $\|\cdot\|$ stands for the Euclidian norm in $\mathbb{R}^{m}$, such that:

- the uniform finite time convergence property holds;

- there exists a continuous function $\rho_{\varepsilon, K}: \mathbb{R}^{n} \rightarrow \mathbb{R}$ satisfying (9) for $\rho=\rho_{\varepsilon, K}$, such that, for every $\left(x_{0}, s_{0}\right) \in K \times \mathcal{N}$, all functions $e, d$ satisfying the regularity assumptions (4) and inequalities (10) for $\rho=\rho_{\varepsilon, K}$, the maximal solution of $\mathcal{H}_{(e, d)}$ starting from $\left(x_{0}, s_{0}\right)$ reaches $O$ within time $T\left(x_{0}\right)+\varepsilon$, where $T\left(x_{0}\right)$ denotes the minimal time to steer the system (3) from $x_{0}$ to 0 , under the constraint $\|u\| \leq 1$.

\section{Main result}

Theorem 2.1: Assume that Hörmander's condition holds. If there exists no nontrivial singular minimal time solution of (1), (2), starting from 0 , then the origin is a semi-global minimal time hybrid robust stabilizable equilibrium for the system (1), under the constraint (2).

Remark 2.3: The problem of global robust minimal time stabilization (i.e. $K=\mathbb{R}^{n}$ in Definition 2.7) cannot be achieved a priori because measurement noises may then accumulate and slow down the solution reaching 0 (compare with [9]).

Remark 2.4: The assumption of the absence of nontrivial singular minimizing solutions is classical in optimal control theory. Notice the following facts.

- If $m \geq n$, then there exists no singular solution.

- Denote by $\mathcal{F}_{m}$ the set of $m$-uples of linearly independent vector fields $\left(f_{1}, \ldots, f_{m}\right)$, endowed with the $C^{\infty}$ Whitney topology. If $m \geq 3$, there exists an open dense subset of $\mathcal{F}_{m}$, such that any control system of the form (1), associated to a $m$-tuple of this subset, admits no nontrivial singular minimizing solution (see [11], [12], see also [2] for the existence of a dense set only).

- If there exist singular minimizing solutions, then the conclusion on subanalyticity of the function $T$ may fail, and we cannot a priori prove that the set $\mathcal{S}$ of singularities of $T$ is a stratifiable manifold, which is the crucial fact in order to define a hybrid strategy.

\section{SKETCH OF PROOF}

Due to the space limitation, we only sketch the proof (for a complete proof, see [32]).

In Subsection III-A, we study the minimal time control problem with the Hörmander's condition and we define an optimal control law $k^{o p t}$, which is smooth on $\mathbb{R}^{n}$ except on the so-called cut locus set. Thus, outside of any given $\Omega$ neighborhood of this cut locus, we have a natural robustness property.

In Subsection III-B, we define the components of the hysteresis. First, using the minimal time function, the optimal controller is defined, outside a singular set which is a stratified submanifold of codimension greater than or equal to one. Then, the second component of the hysteresis is defined; it consists of a set of controllers, defined in a neighborhood of the singular set.

The main part of the proof, not described here, actually consists in uniting these controllers using an adapted hysteresis strategy, and describing the properties of the closed-loop system with this hybrid feedback law.

\section{A. The optimal controller}

We first interpret the minimal time control problem for the system (1) with the constraint (2) as a sub-Riemannian problem. 
1) Sub-Riemannian distance: Recall that the subRiemannian distance (also called Carnot-Carathéodory distance) is defined as follows in $\mathbb{R}^{n}$ (see [6]). Let $m$ an integer such that $1 \leq m \leq n$, and $f_{1}, \ldots, f_{m}$ be smooth vector fields on $\mathbb{R}^{n}$. For all $x \in \mathbb{R}^{n}$ and $v \in \mathbb{R}^{n}$, set

$g(x, v):=\inf \left\{\sum_{i=1}^{m} u_{i}^{2} \mid u_{1}, \ldots, u_{m} \in \mathbb{R}, \sum_{i=1}^{m} u_{i} f_{i}(x)=v\right\}$.

Then $g(x, \cdot)$ is a positive definite quadratic form on the subspace spanned by $f_{1}(x), \ldots, f_{m}(x)$. Outside this subspace we set $g(x, v)=+\infty$. The form $g$ is called $s u b$ Riemannian metric associated to the $m$-tuple of vector fields $\left(f_{1}, \ldots, f_{m}\right)$. Let $\mathcal{A} C\left([0,1], \mathbb{R}^{n}\right)$ denote the set of absolutely continuous paths in $\mathbb{R}^{n}$ defined on $[0,1]$. Define the length of $\gamma \in \mathcal{A} C\left([0,1], \mathbb{R}^{n}\right)$ as $l(\gamma)=\int_{0}^{1} \sqrt{g(\gamma(t), \dot{\gamma}(t))} d t$. Note that the length of a path does not depend on its parametrization.

Recall that Hörmander's condition holds if the Lie algebra spanned by the vector fields $f_{1}, \ldots, f_{m}$, is equal to $\mathbb{R}^{n}$ at every point $x$. It is well-known that under this condition any two points of $\mathbb{R}^{n}$ can be joined by an absolutely continuous path with finite length.

Definition 3.1: The sub-Riemannian distance associated to the $m$-tuple of vector fields $\left(f_{1}, \ldots, f_{m}\right)$, between two points $x_{0}, x_{1}$, is defined by

$$
\begin{array}{r}
d_{S R}\left(x_{0}, x_{1}\right)=\inf \left\{l(\gamma) \mid \gamma \in \mathcal{A} C\left([0,1], \mathbb{R}^{n}\right),\right. \\
\left.\gamma(0)=x_{0}, \gamma(1)=x_{1}\right\} .
\end{array}
$$

A path $\gamma \in \mathcal{A} C\left([0,1], \mathbb{R}^{n}\right)$ is said to be minimizing if it realizes the sub-Riemannian distance between its extremities.

Remark 3.1: If Hörmander's condition holds, then any two points can be joined by a minimizing path, and the topology defined by the sub-Riemannian distance $d_{S R}$ coincides with the usual topology of $\mathbb{R}^{n}$.

2) Optimal control formulation: Since the length of a path does not depend on its parametrization, if a path $x(\cdot)$ : $[0, T] \rightarrow \mathbb{R}^{n}$ is parametrized by its arc-length, then there holds almost everywhere on $[0, T]$

$$
\dot{x}(t)=\sum_{i=1}^{m} u_{i}(t) f_{i}(x(t)), \sum_{i=1}^{m} u_{i}(t)^{2}=1,
$$

and the length of $x(\cdot)$ is equal to $T$. Therefore, the subRiemannian problem is equivalent to the minimal time problem for the system (1) with the (non convex) constraint $\sum_{i=1}^{m} u_{i}^{2}=1$. Obviously, it is also equivalent to the minimal time problem for the system (1) with the (convex) constraint (2). We sum up the situation in the following result.

Lemma 3.1: The minimal time problem for the system (1) with the constraint (2) is equivalent to the subRiemannian problem associated to the $m$-tuple of vector fields $\left(f_{1}, \ldots, f_{m}\right)$. Moreover, the minimal time $T(x)$ needed to steer the system (1) with the constraint (2) from 0 to a point $x \in \mathbb{R}^{n}$ is equal to the sub-Riemannian distance $d_{S R}(0, x)$ of $x$ to 0 .

In particular, minimal time solutions of (1), (2), are exactly minimizing paths of the previous sub-Riemannian problem.
This equivalence permits to work in the framework of subRiemannian geometry.

Singular solutions (resp. singular controls) are defined in the same way as previously (see Definition 2.2).

3) Computation of minimizing solutions: Let $x \in \mathbb{R}^{n}$. The sub-Riemannian problem of determining a minimizing solution steering 0 to $x$ can be easily seen (up to reparametrization, and using the Cauchy-Schwarz inequality) to be equivalent to the optimal control problem of finding a control $u(\cdot) \in \mathcal{U}$ such that the solution of the control system (1) steers 0 to $x$ in time 1 , and minimizes the cost function

$$
C(u(\cdot))=\int_{0}^{1} \sum_{i=1}^{m} u_{i}(t)^{2} d t .
$$

If a control $u(\cdot)$ associated to a solution $x(\cdot)$ so that $x(0)=0$ is optimal, then there exists a nontrivial Lagrange multiplier $\left(\psi, \psi^{0}\right) \in \mathbb{R}^{n} \times \mathbb{R}$ such that

$$
\psi \cdot d E_{1}(u(\cdot))=-\psi^{0} d C(u(\cdot)),
$$

where $d E_{1}(u(\cdot))$ (resp. $\left.d C(u(\cdot))\right)$ denotes the Fréchet derivative of $E_{1}$ (resp. $C$ ) at the point $u(\cdot)$. Moreover, according to Pontryagin's maximum principle (see [27]), the solution $x(\cdot)$ is the projection of an extremal, that is a quadruple $\left(x(\cdot), p(\cdot), p^{0}, u(\cdot)\right)$, solution of the constrained Hamiltonian system

$$
\begin{gathered}
\dot{x}(t)=\frac{\partial H}{\partial p}\left(x(t), p(t), p^{0}, u(t)\right), \\
\dot{p}(t)=-\frac{\partial H}{\partial x}\left(x(t), p(t), p^{0}, u(t)\right), \\
\frac{\partial H}{\partial u}\left(x(t), p(t), p^{0}, u(t)\right)=0,
\end{gathered}
$$

almost everywhere on $[0,1]$, where

$$
H\left(x, p, p^{0}, u\right)=\left\langle p, \sum_{i=1}^{m} u_{i} f_{i}(x)\right\rangle+p^{0} \sum_{i=1}^{m} u_{i}^{2}
$$

is the Hamiltonian of the optimal control problem, $p(\cdot)$ (called adjoint vector) is an absolutely continuous mapping on $[0,1]$ such that $p(t) \in \mathbb{R}^{n}$, and $p^{0}$ is a real nonpositive constant. Moreover, there holds $\left(p(1), p^{0}\right)=\left(\psi, \psi^{0}\right)$, up to a multiplying scalar. If $p^{0}<0$ then the extremal is said to be normal, and in this case it is normalized to $p^{0}=-1 / 2$. If $p^{0}=0$ then the extremal is said to be abnormal.

Remark 3.2: Any singular solution is the projection of an abnormal extremal, and conversely.

Using the previous normalization, controls associated to normal extremals can be computed as

$$
u_{i}(t)=\left\langle p(t), f_{i}(x(t)\rangle, i=1, \ldots, m .\right.
$$

Hence, normal extremals are solutions of the Hamiltonian system

$$
\dot{x}(t)=\frac{\partial H_{1}}{\partial p}(x(t), p(t)), \dot{p}(t)=-\frac{\partial H_{1}}{\partial x}(x(t), p(t)),
$$

where

$$
H_{1}(x, p)=\frac{1}{2} \sum_{i=1}^{m}\left\langle p, f_{i}(x)\right\rangle^{2}
$$




\section{4) The cut locus:}

Definition 3.2: A point $x \in \mathbb{R}^{n}$ is not a cut point if there exists a minimizing solution joining 0 to $x$, which is the strict restriction of a minimizing solution starting from 0 . The $c u t$ locus, denoted by $\mathcal{L}$, is the set of all cut points.

In other words, a cut point is a point at which a minimizing solution ceases to be optimal.

The following result on the cut locus is crucial (see [32] for a proof).

Proposition 3.2: Assume that the $m$ vector fields $f_{1}, \ldots, f_{m}$ are analytic, and that there exists no singular minimizing solution starting from 0 . Then, the set of points where the sub-Riemannian distance to 0 is not analytic is equal to the cut locus, that is, $\operatorname{Sing} d_{S R}(0, \cdot)=\mathcal{L}$.

Proposition 3.2 provides the key argument of the proof. Indeed, on the one part, in the absence of nontrivial minimizing solutions, the mapping $d_{S R}(0, \cdot)$ is subanalytic outside 0 , and in particular its singular set $\mathcal{S}:=\operatorname{Sing}\left(d_{S R}(0, \cdot)\right)$ is a subanalytic manifold of codimension greater than or equal to one. Outside this singular set, this feedback law is analytic and defines our feedback law $k^{o p t}$.

\section{B. Components of the hysteresis, and hybrid strategy}

The function $T(\cdot)$, which coincides with the function $d_{S R}(0, \cdot)$, is subanalytic outside 0 , and hence, its singular set $\mathcal{S}$ (i.e., the analytic singular support of $T(\cdot)$ ) is a stratified submanifold of $\mathbb{R}^{n}$, of codimension greater than or equal to 1. The objective is then to construct neighborhoods of $\mathcal{S} \backslash\{0\}$ in $\mathbb{R}^{n}$ whose complements share invariance properties for the optimal flow.

1) The optimal controller: Outside the singular set $\mathcal{S}$, the function $T(\cdot)$ is analytic, and the minimal time controllers steering a point $x \in \mathbb{R}^{n} \backslash \mathcal{S}$ to 0 are given by the closed-loop formula

$$
u_{i}(x)=-\frac{1}{2}\left\langle\nabla\left(T(x)^{2}\right), f_{i}(x)\right\rangle, i=1, \ldots, m .
$$

The smoothness of this optimal controller outside the submanifold $\mathcal{S}$ ensures a robustness property of the stability outside $\mathcal{S}$. The following lemma, yieldings invariance properties of the optimal flow, is proved in [32].

Lemma 3.3: For every neighborhood $\Omega$ of $\mathcal{S} \backslash\{\bar{x}\}$ in $\mathbb{R}^{n}$, there exists a neighborhood $\Omega^{\prime}$ of $\mathcal{S} \backslash\{\bar{x}\}$ in $\mathbb{R}^{n}$, satisfying

$$
\Omega^{\prime} \subsetneq \operatorname{clos}\left(\Omega^{\prime}\right) \subsetneq \Omega
$$

such that every trajectory of the closed-loop system (1) with the optimal controller, joins a point $x \in \mathbb{R}^{n} \backslash \Omega$ to $\bar{x}$, and is contained in $\mathbb{R}^{n} \backslash \Omega^{\prime}$.

A switching strategy must be defined in order to connect the optimal controller to other controllers, defined next, which have to be continuous in the neighborhood $\Omega^{\prime}$ of the submanifold $\mathcal{S}$. The switching strategy is achieved by adding a dynamical discrete variable $s_{d}$ and using a hybrid feedback law.

Robustness properties of the system in closed-loop with the optimal controller are investigated in [32].
2) The second component of the hysteresis: The second component of the hysteresis consists of a set of controllers, defined in a neighborhood of $\mathcal{S}$. Since $\mathcal{S}$ is a stratified submanifold of $\mathbb{R}^{n}$ of codimension greater than or equal to one, there exists a partition $\left(M_{i}\right)_{i \in \mathbb{N}}$ of $\mathcal{S}$, where $M_{i}$ is a stratum, i.e., a locally closed submanifold of $\mathbb{R}^{n}$.

\section{End of the proof}

To conclude the proof, we unit have the optimal controller together with the second component of the hysteresis defined previously, using an hysteresis. Note that, it is proved in [33] that this class of feedbacks has natural generic robustness properties with respect to measurement, actuator noise and external disturbances. It is also the case of our hybrid feedback. However, since, in our context, we are interested in a quasi-optimal property, we cannot apply [33] directly. This procedure is similar to the one of [29], where an infinite number of state-feedbacks are united.

In [32], a family of three nested patchy vector fields is required to prove the main result. Some of the patches define the dynamics of the discrete component of the hybrid controller, and the others are used for technical reasons to handle the measurement noises. The precise definition of this hysteresis procedure, so as the description of the properties of the closed-loop system with this hybrid feedback law, actually represent the main part of the proof of the main result (see [32] for details).

Note that this program was achieved in an explicit way in [31]; in particular, in this reference, the hybrid feedback law is completely explicit, and defined in a very simple way. The result of [32], whose proof is sketched here, is rather an existence result, though we explain how to derive an hysteresis feedback law, in function of the Lie bracket configuration of the system. The main result, Theorem 2.1, however points out the main assumption under which it is possible to achieve the stabilization process, namely, the absence of singular minimal time solutions of the control system (1), under the constraint (2).

\section{REFERENCES}

[1] A. Agrachev, Compactness for SR minimizers and subanalyticity, Rend. Sem. Mat. Pol. Univ. Torino 56, 1998.

[2] A. Agrachev, J. P. Gauthier, On subanalyticity of Carnot-Carathéodory distances, Ann. Inst. H. Poincaré Anal. Non Linéaire 18, 3, 2001.

[3] A. Agrachev, Yu. Sachkov, Control theory from the geometric viewpoint, Springer, 2004.

[4] F. Ancona, A. Bressan, Flow stability of patchy vector fields and robust feedback stabilization, SIAM J. Cont. Opt. 41, 5, 2002, pp. 1455-1476.

[5] A. Astolfi, Discontinuous control of the Brockett integrator, European J. Control 4, 1998, pp. 49-53.

[6] A. Bellaïche, Tangent space in sub-Riemannian geometry, in SubRiemannian geometry, Birkhäuser, 1996.

[7] A. Bensoussan, J. L. Menaldi, Hybrid control and dynamic programming, Dyn. Cont. Discrete Impulsive Syst. 3, 4 (1997) 395-442.

[8] M. S. Branicky, Multiple Lyapunov functions and other analysis tools for switched and hybrid systems, IEEE Trans. Automat. Cont. 43 1998, pp. $475-482$.

[9] A. Bressan, Singularities of stabilizing feedbacks, Rend. Sem. Mat. Univ. Pol. Torino 56, 4, 1998, pp. 87-104.

[10] R. W. Brockett, Asymptotic stability and feedback stabilization, in Differential geometric control theory, R. W. Brockett, R. S. Millman and H. J. Sussmann, ed., Boston, Birkhäuser, 1983, pp. 181-191. 
[11] Y. Chitour, F. Jean, E. Trélat, Propriétés génériques des trajectoires singulières, Comptes Rendus Math. 337, 1, 2003, pp. 49-52.

[12] Y. Chitour, F. Jean, E. Trélat, Genericity properties for singular trajectories, Preprint Univ. Paris-Sud, Orsay, 2003, submitted.

[13] F. H. Clarke, Yu. S. Ledyaev, L. Rifford, R. J. Stern, Feedback stabilization and Lyapunov functions, SIAM J. Cont. Opt. 39, 1, 2000, pp. 25-48.

[14] R. T. Closkey, R. M. Murray, Exponential stabilization of driftless nonlinear control systems using homogeneous feedback, IEEE Trans. Automat. Control 42, 5, 1997, pp. 614-628.

[15] J.-M. Coron, Global asymptotic stabilization for controllable systems without drift, Math. Control Signals Syst. 5, 1992, pp. 295-312.

[16] L. van den Dries, C. Miller, Geometric categories and o-minimal structures, Duke Math. Journal 84, 2, 1996.

[17] R. Goebel and A.R. Teel, Solutions to hybrid inclusions via set and graphical convergence with stability theory applications, submitted, 2004.

[18] R. Goebel, J. Hespanha, A.R. Teel, C. Cai and R. Sanfelice, Hybrid systems: generalized solutions and robust stability, IFAC Symp. on Nonlinear Control Systems, Stuttagart, Germany, 2004.

[19] R. M. Hardt, Stratification of real analytic mappings and images, Invent. Math. 28, 1975

[20] H. Hironaka, Subanalytic sets, Number theory, algebraic geometry and commutative algebra, in honor of Y. Akizuki, Tokyo, 1973.

[21] I. Kolmanovsky, N. H. McClamroch, Developments in nonholonomic control problems, IEEE Control Systems 15, 1995, pp 20-36.

[22] Yu. S. Ledyaev, E. D. Sontag, A remark on robust stabilization of general asymptotically controllable systems, Proc. Conf. on Information Sciences and Systems, Johns Hopkins, Baltimore, 1997, pp. 246-251.

[23] E. Litsyn, Y. V. Nepomnyashchikh, A. Ponosov, Stabilization of linear differential systems via hybrid feedback controls, SIAM J. Cont. Opt. 38, 2000, pp. 1468-1480.

[24] P. Morin, J.-B. Pomet, C. Samson, Developments in time-varying feedback stabilization of nonlinear systems, IFAC Symp. on Nonlinear Control System Design, Enschede, The Netherlands 3, 1998.

[25] P. Morin, C. Samson, Practical stabilization of driftless systems on Lie groups: the transverse function approach, IEEE Trans. Automat. Control 48, 9, 2003, pp. 1496-1508.

[26] P. Morin, C. Samson, Exponential stabilization of nonlinear driftless systems with robustness to unmodeled dynamics, ESAIM Cont. Optim. Calc. Var. 4, 1999, pp. 1-35.
[27] L. S. Pontryagin, V. G. Boltyanskij, R. V. Gamkrelidze, E. F. Mishchenko, The mathematical theory of optimal processes, Interscience Publishers, John Wiley \& Sons, New York, 1962.

[28] C. Prieur, Uniting local and global controllers with robustness to vanishing noise, Math. Control Signals Systems 14, 2001, pp. 143 172

[29] C. Prieur, Asymptotic controllability and robust asymptotic stabizability, SIAM J. Cont. Opt., 43, 5, 2005, pp. 1888-1912.

[30] C. Prieur, A. Astolfi, Robust stabilization of chained systems via hybrid control, IEEE Trans. Automat. Control 48, 10, 2003, pp. 1768-1772.

[31] C. Prieur, E. Trélat, Robust optimal stabilization of the Brocket integrator via a hybrid feedback, Math. Control Signals Syst., 17, 3, 2005, pp. 201-216.

[32] C. Prieur, E. Trélat, Quasi-optimal robust stabilization of control systems, Preprint, 2005.

[33] C. Prieur, R. Goebel, A. Teel, Results on robust stabilization of asymptotically controllable systems by hybrid feedback, IEEE Conf. on Dec. and Cont. and Eur. Cont. Conf. (CDC-ECC'05) Seville, Spain, 2005.

[34] L. Rifford, The stabilization problem: AGAS and SRS feedbacks, in Optimal Control, Stabilization, and Nonsmooth Analysis, Lectures Notes in Control and Information Sciences 301, Springer-Verlag, Heidelberg, 2004, pp. 173-184.

[35] E. D. Sontag, Clocks and insensitivity to small measurement errors, ESAIM Cont. Opt. Calc. Var. 4, 1999, pp. 537-557.

[36] E. D. Sontag, Stability and stabilization: Discontinuities and the effect of disturbances, in Nonlinear Analysis, Differential Equations, and Control, Proc. NATO Advanced Study Institute, Montreal, F. H. Clarke, R. J. Stern, ed., Kluwer, 1999, pp. 551-598.

[37] H. J. Sussmann, Subanalytic sets and feedback control, J. Diff. Eq 31, 1, 1979, pp. 31-52.

[38] M. Tamm, Subanalytic sets in the calculus of variation, Acta Math. 146, 1981.

[39] L. Tavernini, Differential automata and their discrete simulators, Nonlinear Anal. 11, 1997, pp. 665-683.

[40] E. Trélat, Etude asymptotique et transcendance de la fonction valeur en contrôle optimal ; catégorie log-exp en géométrie sousriemannienne dans le cas Martinet. $\mathrm{PhD}$, Univ. de Bourgogne, Dijon, France, 2000. 